\section{Ultra Low Voltage BSE Imaging}

Michael D.G. Steigerwald

LEO Electron Microscopy steigerwald@leo.de

The Principle of In-lens Detection

LEO's field emission scanning electron microscopes are all based on the "GEMINI" principle as shown in figure 1. In order to reduce aberrations and sensitivity to interfering stray-fields the electron optical column possesses a positively biased booster that shifts the energy of the primary electrons. The incident beam is focussed by a combination of a magnetic lens with an axial gap that

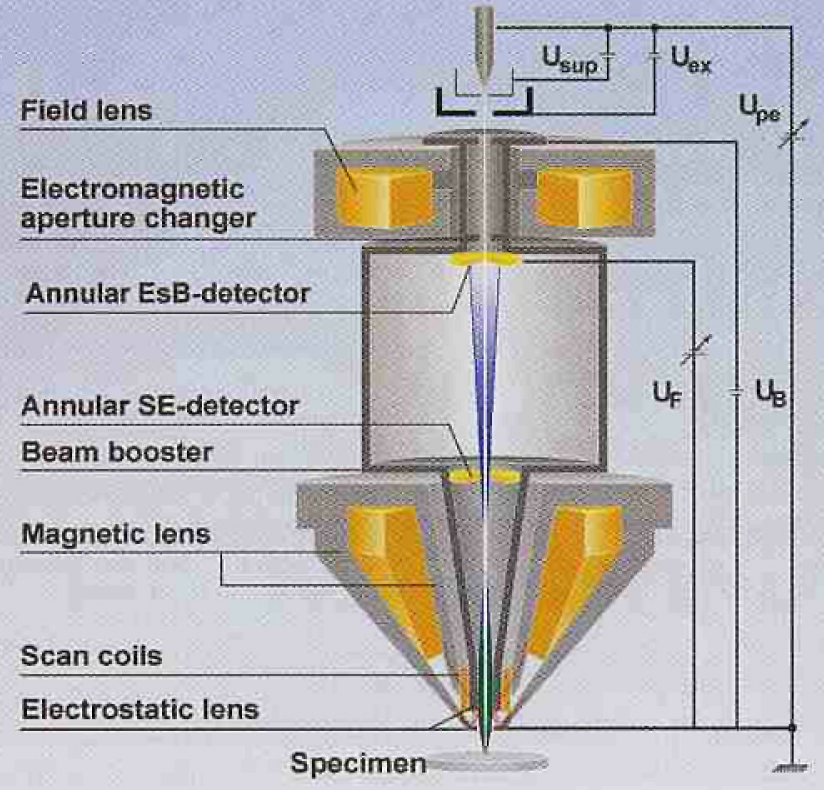

Fig.1: Bias concept of Gemini

avoids field leakage to the specimen and an electrostatic retarding lens formed by the beam booster together with the grounded pole piece cap. Shortly before the electrons hit the specimen they are decelerated down to the desired primary energy. A suitable explanation for the reduction of spherical and chromatic aberrations is that the electron beam is focussed by the objective lens at higher energies and smaller electron beam diameters. Another advantageous effect of this arrangement is the collection of secondary electrons emerging from the sample surface attracted and accelerated by the positively biased electrode of the beam booster and finally projected onto the In-lens detector.

\section{Energy and Angle selective BSE Detection - EsB}

To understand the basic principle of the new detection system, one has to have a closer look at the energy spectra as well as the take-off angle distributions of the released electrons and their trajectories through the electron column. Figure 2 illustrates a schematic energy spectrum of electrons escaping from the specimen. Secondary electrons (green), possessing very low energies by definition, are released from the near surface and carry therefore topographic information, whereas backscattered electrons (blue), which have undergone at least one large angle scattering, originate from larger depths and possess compositional information. Beside electron energy level both electron types also differ in respect to their take-off angle distribution. While the distribution of secondary

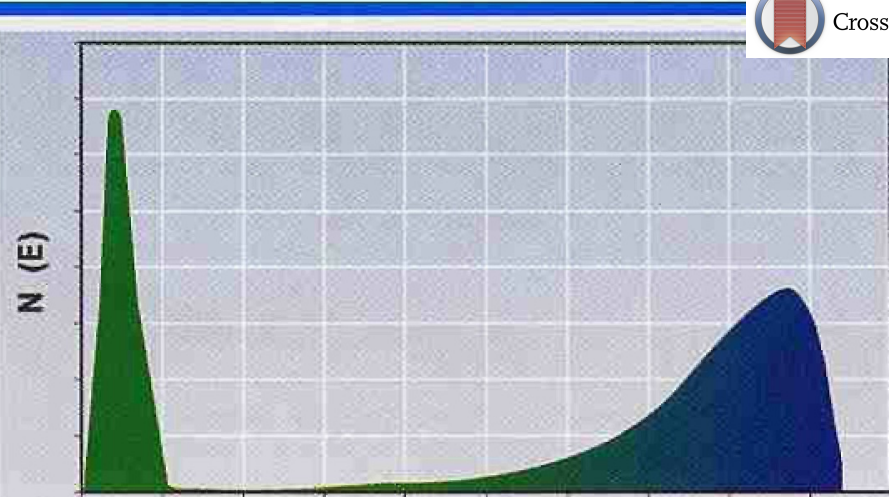

\section{$\mathrm{E} / \mathrm{E}_{0}$}

Fig. 2: Electron energy spectrum

electrons orientates perpendicularly to topographic structures, backscattered electrons emerge form the bulk material and are therefore less sensitive to surface micro-inclinations. After emerging from the specimen surface most of the electrons are attracted by the beam booster and move upstream into the GEMINI column. Because of the chromatic aberration of the magnetic lens, the electrons are forced on different trajectories depending on their energy when passing the focussing field.

Both, the deflection of the lens and the different take-off angle distribution result in different phase spaces at the position of the lower annular In-lens detector (Figure 3 ).

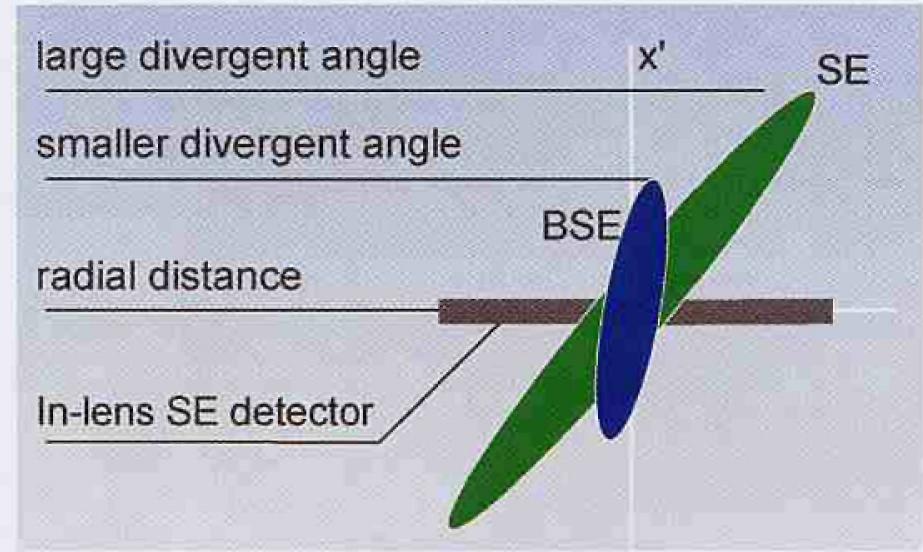

Fig.3: Phases space comparison

The green ellipse indicates secondary electrons possessing large divergence and a wide spatial spread, whereas the backscattered electron emittance is significantly smaller resulting in an effective separation of secondary and backscattered electrons at the position of the lower In-lens detector. As a consequence of different phase spaces, backscattered electrons have a closer radial distance in comparison to secondaries and transit through the central aperture of the In-lens secondary detector, while secondary electrons land on the In-lens detector and are collected.

From figure 4 depicting the radial distribution and the dimension of the detector aperture (red line) it is clear that for optimized conditions a filtering efficiency of $90 \%$ may be achieved by applying the method of "Energy and Angle selective BSE detection". Electrons passing the lower In-lens detector may be collected at the upper "EsB" detector. These are mainly the so-called "high angle" backscattered electrons, but since there are still some secondary electrons inside the phase space volume of the backscattered they are not collected at the lower In-lens detector. This means that the 


\section{Thermal Microscopy Solutions}

From McCrone

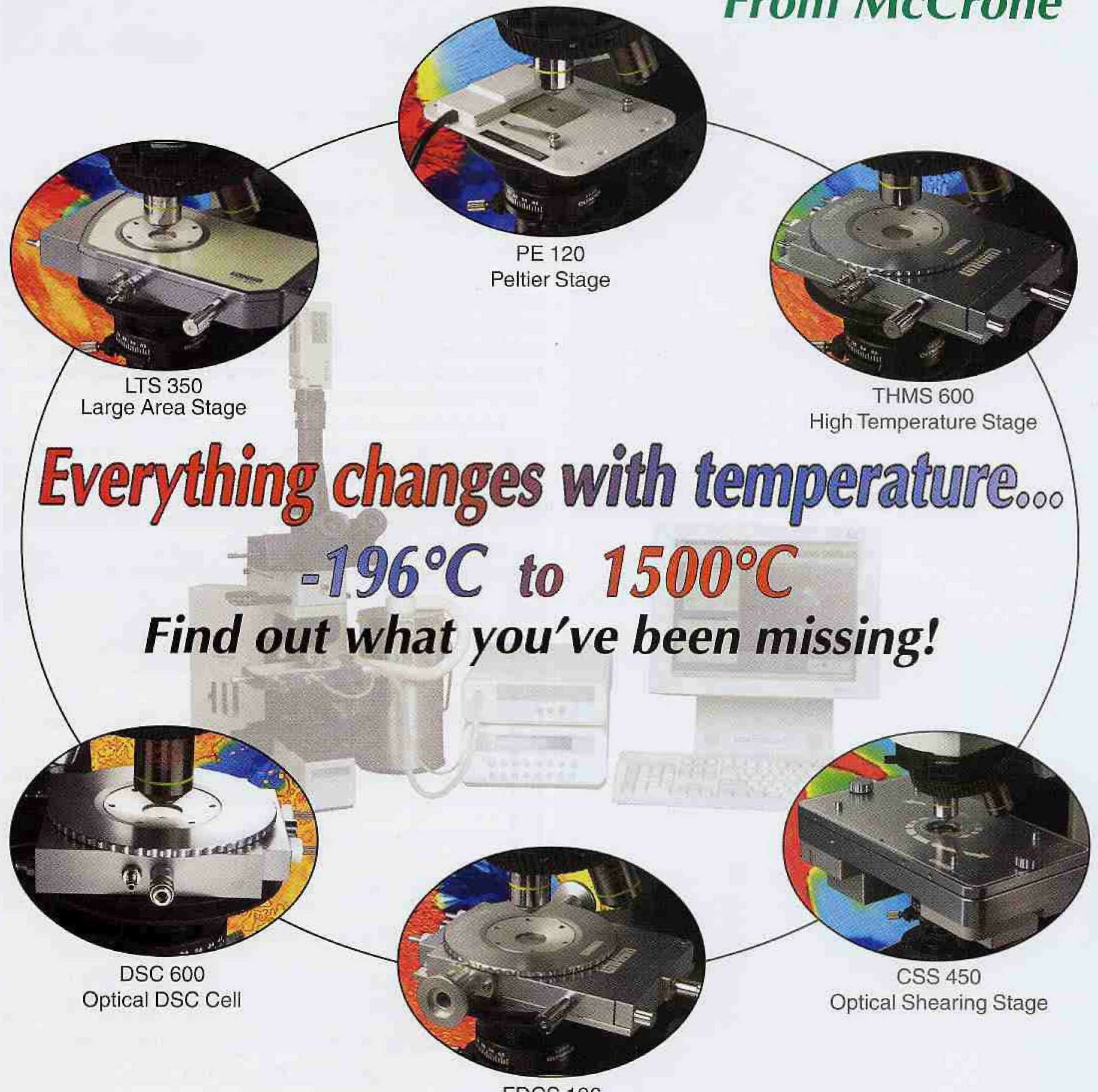

FDCS 196

Freeze Dry Stage

McCrone Microscopes \& Accessories Authorized Linkam Thermal Stage Dealer

Announcing Modern
Microscopy

Featuring Periodic Articles, Reviews and Tutorials about Microscopy by some of the most Experienced Researchers in the Industry. 


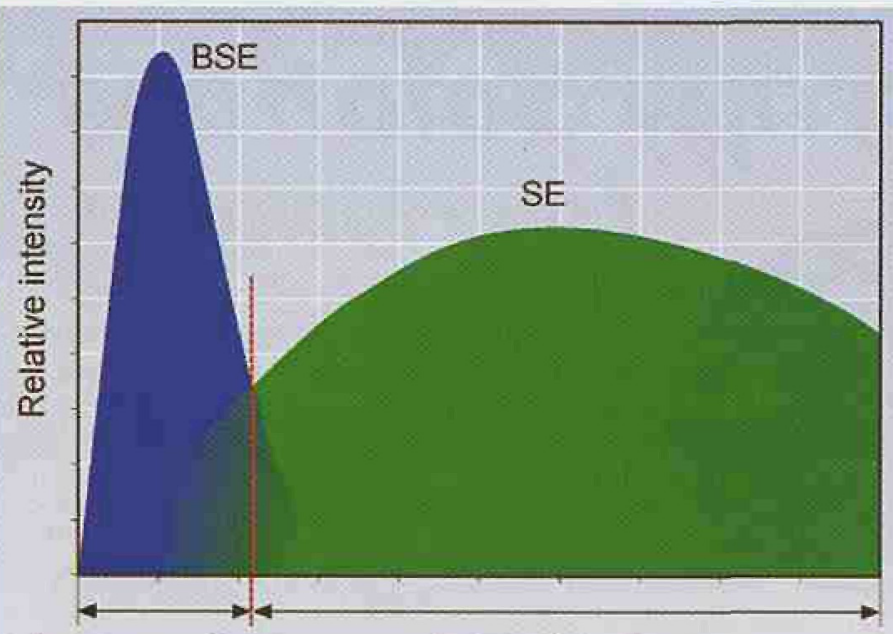

In-lens aperture In-lens detector area

Fig.4: Radial distribution

compositional information of the EsB detector is superimposed by undesirable surface signal. To remove this undesirable signal a negatively biased filtering grid is installed below the EsB detector to repel the secondary electrons. Adjustment of the filtering grid is in the range from 0 to minus $3000 \mathrm{~V}$ enabling real-time mixture of surface, voltage, and material contrasts. The momentary obtained energy resolution is in the range of $120 \mathrm{eV}$, allowing resolution enhancement in the BSE signal. The potential on the filtering grid does not interfere with the profile of the high resolution primary electron beam. The combination of detector geometry and the filter voltage enables simultaneous observation of the In-lens secondary and the EsB detector signals. Below 3000 V BSE filtering has been realized.

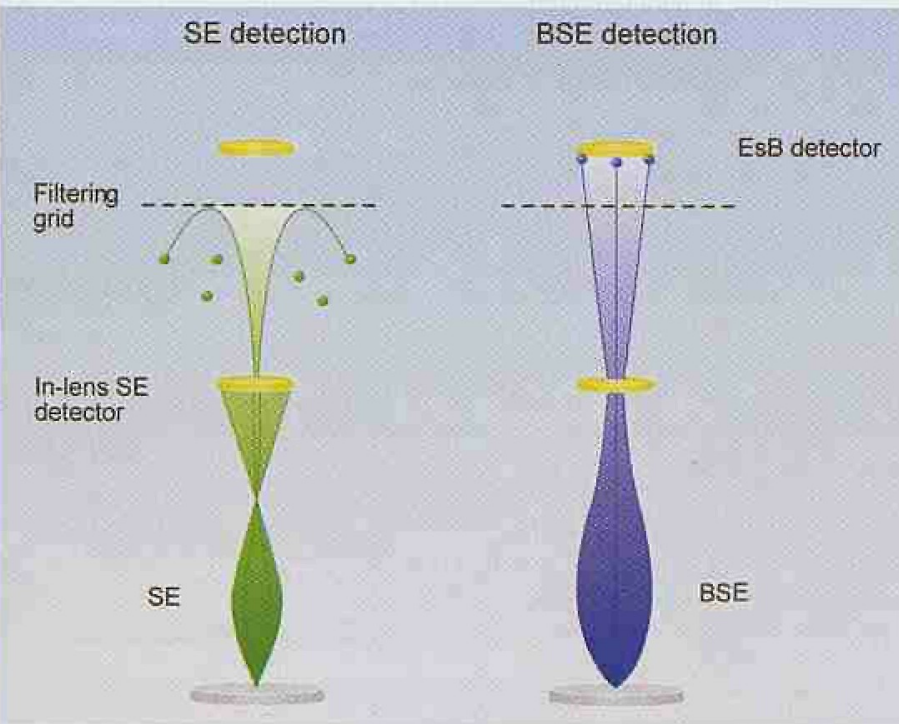

Fig.5: The SEs (green) are projected onto the lower In-lens detector and the BSES (b/ue) are guided onto the upper ESB detector

Figure 5 shows clearly the beam shapes of both electron types and the general functioning of EsB detection and filtering.

\section{Results}

Figure 6 depicts the advantages of the new detector arrangement. While the upper image clearly shows topographical and voltage information, the lower micrograph pronounces compositional contrast and suppresses any charging or edge emphasis effects allowing for accurate metrology to be performed.

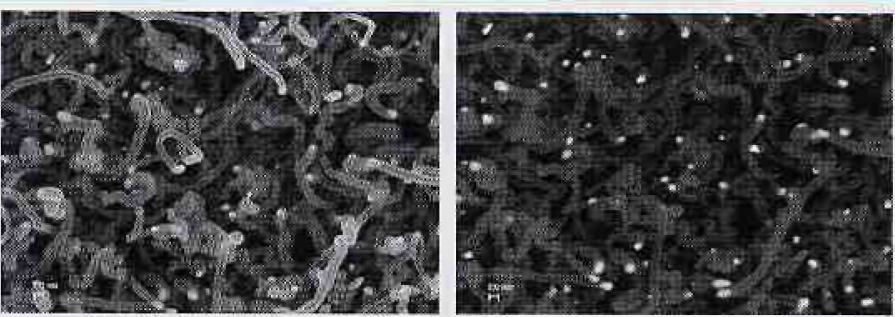

Fig.6: Nanotube sample at $4 \mathrm{kV}$

Left: SE image. Right: BSE image with ESB detector (filtering voitage $600 \mathrm{eV}$ ). Image courtesy of Dr. Heiner Jacksch LEO Electron Microscopy Group.

\section{Conclusions}

The new EsB detector shows excellent results at low beam energy and very short working distances (100V@1mm WD) allowing ultra high resolution and precise imaging. The integrated EsB detector does not require any additional adjustments, does not interfere with the primary electron beam and enables simultaneous real time imaging and mixing of SE and BSE signals. The direct detection method of the EsB detector has proven to be very effective. The detection efficiency is close to $100 \%$ against the expected $50 \%$ of conversion plate type detectors.

Compared to a chamber mounted BSE detector the EsB detector, which only detects the "high angle" BSE, delivers higher resolution at shorter working distances enabling high precision feature measurements.

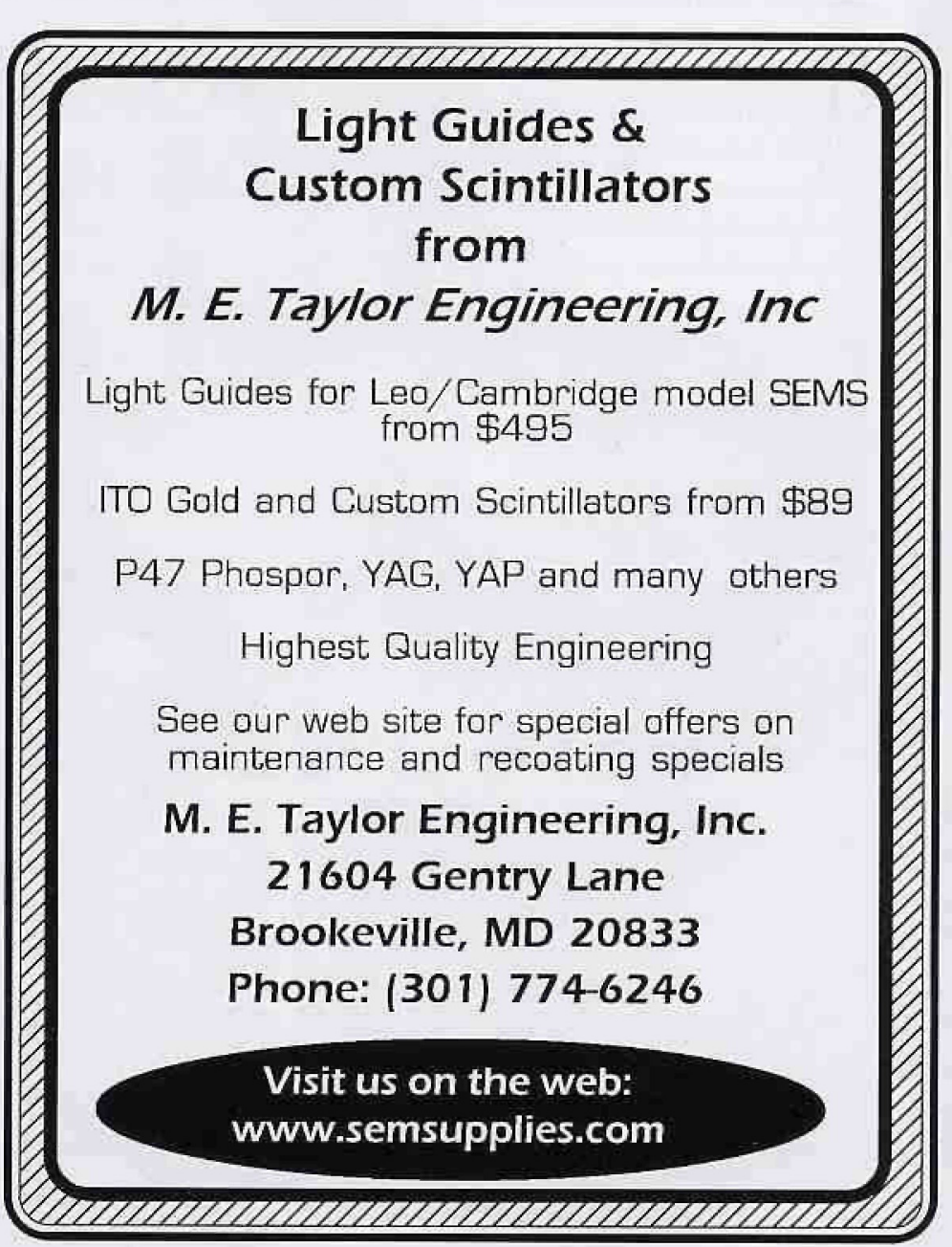




\section{Visionary \\ microanalysis technology See it today}

Available right now with:

Unique automatic identification of all elements

Simple image assisted set-up

Fast feature detection and data collection algorithm

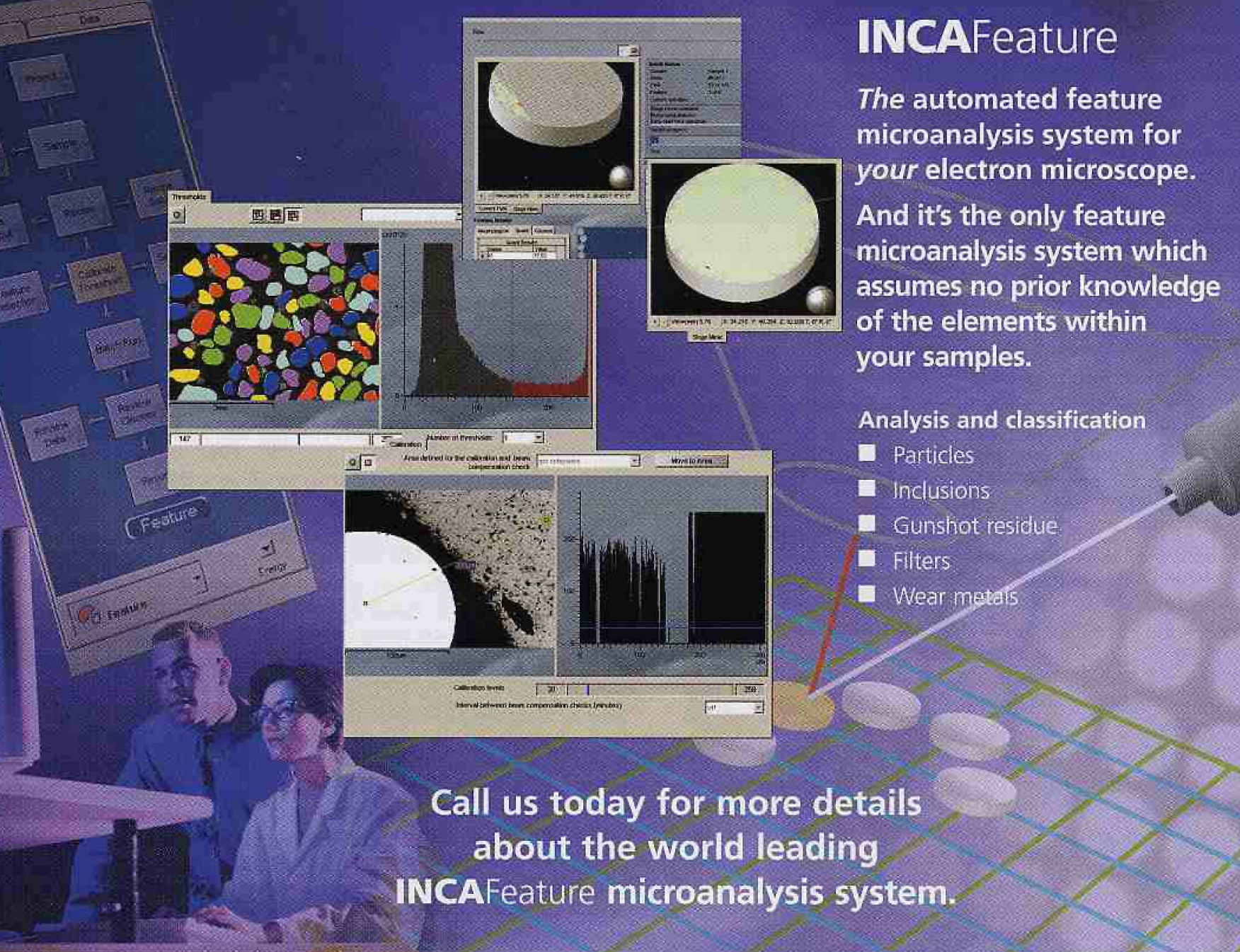

